\title{
Stochastic Simulation and Application of Monthly Rainfall and Evaporation
}

\author{
Nana Han and Yang-ren Wang \\ Department of Hydraulic Engineering, Tianjin Agricultural University, Tianjin 300384 \\ $\{$ hnn23144, wyrf $@ 163$.com
}

\begin{abstract}
Statistic was done for the precipitation and evaporation monitoring data of Yuncheng from 1971 to 2007. The first-order seasonal autoregressive models were set up, considering separately for the normal and skewed distribution of rainfall and evaporation. Long series of monthly precipitation and evaporation sequences were generated. Compared the average, standard deviation and other parameters of simulation sequences with measured sequence, the results of skewed simulation had better agreement with the measured one. Then it proved that skewness model maintains the main statistical characteristics of the measured sequence. 20 groups of equal length with the measured sequence of precipitation and evaporation sequence could be generated through the skewness model. The sequence of irrigation water of the winter wheat multiple corns planting type had been calculated, using the " $\alpha$ " value method. And comparative analysis with the measured result was performed. The simulation computed result was found in good coincidence with the measure computed result.
\end{abstract}

Keywords: precipitation, evaporation, seasonal autoregressive model, irrigation water.

\section{Introduction}

Crop irrigation water can be determined by the field water balance method, using the precipitation and evaporation data. Based on the long series of data, the scale of irrigation projects can be determined. Thus many of the data needed, actually the measured data were limited. Aiming at the randomicity of precipitation and evaporation, the precipitation and evaporation were randomly treated, and simulated using random hydrology methods. Then Long series of irrigation water sequence can be calculated in order to develop irrigation schemes more in line with actual crop water demand. Azhar [1] considered evapotranspiration, precipitation randomness, introduced a minimum of irrigation water requirement of rice cumulative probability distribution, and used to guide irrigated rice irrigation. Huan-Jie Cai [2] simulated the daily precipitation and studied the irrigation problems of wheat and maize. Zuo Xiaoxia [3] generated long series of precipitation and evaporation by the stochastic hydrology method. Then a long series of rice irrigation process were calculated using the cross-correlation of rainfall evaporation. Wen Ji [4] simulated the evapotranspiration, 
and the results derived stochastic simulation model of winter wheat irrigation. Zai Song-mei [5] simulated the precipitation and evapotranspiration using random hydrology methods based on time-series, and then the simulated values were used into the water balance equation to determine the irrigation time and amount. Wen Ji [6] analyzed the stochastic simulation techniques of crop irrigation and pointed out that it has strong universality. This paper simulated the sequences of precipitation and evaporation by the seasonal autoregressive model, and calculated the sequence of crop irrigation water in Yuncheng.

\section{$2 \quad$ Materials and Methods}

\subsection{Model}

Seasonal autoregressive model is used to simulate monthly precipitation and evaporation. The general form of seasonal autoregressive model is as follows [7]:

$$
z_{t, \tau}=\varphi_{1, \tau} z_{t, \tau-1}+\varphi_{2, \tau} z_{t, \tau-2}+\ldots+\varphi_{p, \tau} z_{t, \tau-p}+\varepsilon_{t, \tau}
$$

Where $Z_{t, \tau}$ is standardized sequence, $\varphi_{1, \tau} 、 \varphi_{2, \tau} 、 \ldots \varphi_{p, \tau}$ are respectively the auto regression coefficients of the $\tau$ season from 1 to $p$ orders, and $\varepsilon_{t, \tau}$ is the independent random sequences of the t year of the $\tau$ season.

Standardized sequence which is to eliminate the seasonal effects of average、 variance can be obtained by standardizing the hydrological series. Formula is as follows.

$$
z_{t, \tau}=\frac{x_{t, \tau}-u_{\tau}}{\sigma_{\tau}}
$$

Where $u_{\tau}$ is average, $\sigma_{\tau}$ is standard deviation, and $x_{t, \tau}$ is seasonal hydrological sequence $(\mathrm{t}=1,2, \ldots, \mathrm{n}, \mathrm{n}$ is the number of years ; $\tau=1,2, \ldots, w, w$ is the number of seasons), which can be represented through matrix.

$$
\left[\begin{array}{ccccc}
x_{1,1} & x_{1,2} & x_{1,3} & \ldots & x_{1, w} \\
\vdots & \vdots & \vdots & \vdots & \vdots \\
x_{t, 1} & x_{t, 2} & x_{t, 3} & \ldots & x_{t, w} \\
\vdots & \vdots & \vdots & \vdots & \vdots \\
x_{n, 1} & x_{n, 2} & x_{n, 3} & \ldots & x_{n, w}
\end{array}\right]=\left\{x_{t, \tau}\right\}_{n \times w}
$$


Studies show that a first or second order model will be able to meet the requirements. Thus this article uses a first order seasonal autoregressive model. When $\mathrm{p}=1$, the model is as follows.

$$
z_{t, \tau}=\varphi_{1, \tau} z_{t, \tau-1}+\varepsilon_{t, \tau}
$$

\subsection{Estimation of Model's Parameters}

Parameters of a first order seasonal autoregressive model are $u_{\tau} 、 \sigma_{\tau}^{2} 、 \varphi_{1, \tau}$ 、 and $\sigma_{\varepsilon, \tau}^{2}(\tau=1,2, \ldots w)$. Estimate of $u_{\tau}$ and $\sigma_{\tau}^{2}$ can be separately reached with the average $\left(\bar{x}_{\tau}\right)$ and variance $\left(s_{\tau}^{2}\right)$ of the sample.

$$
\begin{gathered}
\hat{u}_{\tau}=\bar{x}_{\tau}=\frac{1}{n} \sum_{t=1}^{n} x_{t, \tau} \\
\hat{\sigma}_{\tau}^{2}=s_{\tau}^{2}=\frac{1}{n-1} \sum_{t=1}^{n}\left(x_{t, \tau}-\bar{x}_{\tau}\right)^{2} \\
\varphi_{1, \tau}=\hat{\rho}_{1, \tau}=r_{1, \tau}=\frac{\sum_{t=1}^{n}\left(x_{t, \tau}-\bar{x}_{\tau}\right)\left(x_{t, \tau-1}-\bar{x}_{\tau-1}\right)}{(n-1) s_{\tau} s_{\tau-1}}
\end{gathered}
$$

Where $r_{1, \tau}$ is the $\tau$ season's first-order sample autocorrelation coefficient of the $x_{t, \tau}$ sequence, which indicates the linear correlation between the $\tau$ and $\tau-1$ season.

If the sequences of precipitation and evaporation are considered normal one, then the independent random sequence $\varepsilon_{t, \tau}$ is pure normal distribution. The formula is as follows.

$$
\begin{gathered}
\varepsilon_{t, \tau}=\sigma_{\varepsilon, \tau} \xi_{t, \tau} \\
\sigma_{\varepsilon, \tau}=\sqrt{1-r_{1, \tau}^{2}} \\
\xi_{\tau}=\sqrt{-2 \ln u_{1, \tau}} \cos 2 \pi u_{2, \tau}
\end{gathered}
$$

Where $u_{1, \tau}$ and $u_{2, \tau}$ are uniformly distributed random numbers, and $\xi_{\tau}$ is the independent standard normal pure random sequence, namely $\xi_{\tau} \sim N(0,1)$. $\xi_{t, \tau}$ can be obtained by a lot of $u_{t, \tau}$. 
If the sequences of precipitation and evaporation are considered skewed one, then the independent random sequence $\varepsilon_{t, \tau}$ is skewed distribution. In hydrology and water resources, the distribution of $\varepsilon_{t, \tau}$ more considers is a P-III type one. The formula is as follows.

$$
\begin{gathered}
\mathcal{E}_{t, \tau}=\sigma_{\varepsilon, \tau} \Phi_{t, \tau} \\
\Phi_{t, \tau}=\frac{2}{C_{S_{\Phi, \tau}}}\left(1+\frac{C_{S_{\Phi, \tau}} \xi_{t, \tau}}{6}-\frac{C_{S_{\Phi, \tau}}^{2}}{36}\right)^{3}-\frac{2}{C_{S_{\Phi, \tau}}} \\
C_{S_{\Phi, \tau}}=\frac{C_{S_{x, \tau}}-\varphi_{1, \tau}^{3} C_{S_{x, \tau-1}}}{\left(1-\varphi_{1, \tau} r_{1, \tau}\right)^{3 / 2}} \\
C_{S_{x, \tau}}=\frac{1}{n-3} \frac{\sum_{t=1}^{n}\left(x_{t, \tau}-\bar{x}_{\tau}\right)^{3}}{s_{\tau}^{3}}
\end{gathered}
$$

Where $\Phi_{t, \tau}$ is standardized P-III distributed random variable, $C_{S_{\Phi, \tau}}$ is coefficient of skewness of $\Phi_{t, \tau}$, and $C_{S_{x, \tau}}$ is coefficient of skewness of $x_{t, \tau}$.

\subsection{Calculation of Irrigation Water}

Irrigation water is the required amount of water of irrigated land, which is drawn from the source. It depends on the factors such as the irrigated area, crop cultivation, soils, hydrogeological and meteorological conditions. Farm irrigation water is calculated as follows:

$$
M=\alpha E_{0}-\beta P
$$

Where $M$ is crop irrigation water of the crops' whole growth period (mm), $\alpha$ is water demand coefficient, which determined by the measured data (see table 1), $E_{0}$ is water surface evaporation of the crops' whole growth period $(\mathrm{mm}), \mathrm{P}$ is precipitation of the crops' whole growth period ( $\mathrm{mm})$, and $\beta$ is effective utilization coefficient of precipitation, which determined by relevant information, the value of $\beta$ is 0.9 in the months of $11,12,1-4$,and in other months is 0.8 . 
Table 1. The value of $\alpha$

\begin{tabular}{ccccccccccccc}
\hline month & 1 & 2 & 3 & 4 & 5 & 6 & 7 & 8 & 9 & 10 & 11 & 12 \\
\hline$\alpha$ & 0.4 & 0.552 & 0.665 & 0.722 & 0.74 & 0.743 & 0.75 & 0.83 & 0.73 & 0.397 & 0.593 & 0.462 \\
\hline
\end{tabular}

\section{Results and Discussion}

\subsection{Applicability Analysis of the Model}

Statistic was done for the precipitation and evaporation monitoring data of Yuncheng from 1971 to 2007. Considering separately for the normal and skewed distribution of rainfall and evaporation, this paper set up the first-order seasonal autoregressive models. Then three groups of one hundred length sequences were generated. And thus this paper analyzed the parameters of model and application of model. The results were shown in Table 2 and Table 3.

Table 2. Results of model suitability testing of Yuncheng district's monthly precipitation

\begin{tabular}{|c|c|c|c|c|c|c|c|c|c|c|c|c|c|}
\hline \multirow{2}{*}{\multicolumn{2}{|c|}{ Parameter }} & \multicolumn{12}{|c|}{ monthly precipitation $\bullet \mathrm{mm} \bullet$} \\
\hline & & 1 & 2 & 3 & 4 & 5 & 6 & 7 & 8 & 9 & 10 & 11 & 12 \\
\hline 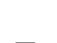 & measured & 6.2 & 7.4 & 17.2 & 35.4 & 46.0 & 64.6 & 106.3 & 83.6 & 99.8 & 50.3 & 23.1 & 6.4 \\
\hline \multirow[t]{3}{*}{$x$} & Simulation (normal) & 6.5 & 7.3 & 17.7 & 34.3 & 45.9 & 66.6 & 104.3 & 84.7 & 88.5 & 50.1 & 22.7 & 6.5 \\
\hline & Simulation (skewness) & 6.1 & 7.6 & 19.2 & 33.4 & 47.0 & 62.9 & 106.2 & 82.3 & 98.1 & 50.1 & 23.1 & 6.9 \\
\hline & Measured & 7.9 & 7.0 & 15.7 & 26.1 & 32.7 & 50.6 & 61.1 & 55.7 & 97.5 & 35.3 & 30.6 & 7.5 \\
\hline \multirow[t]{3}{*}{$\sigma_{\tau}$} & Simulation (normal) & 8.2 & 6.9 & 17.0 & 26.2 & 31.3 & 50.4 & 51.7 & 58.0 & 107.3 & 33.8 & 31.2 & 7.2 \\
\hline & Simulation (skewness) & 7.3 & 6.7 & 15.9 & 24.6 & 34.4 & 49.2 & 54.2 & 50.6 & 91.8 & 34.6 & 29.1 & 7.1 \\
\hline & Measured & 2.044 & 1.148 & 1.215 & 1.451 & 0.868 & 2.600 & 0.835 & 1.286 & 3.176 & 0.789 & 2.578 & 0.998 \\
\hline \multirow[t]{3}{*}{$\mathrm{Cs}$} & Simulation (normal) & 0.103 & 0.022 & 0.129 & 0.147 & 0.054 & 0.002 & -0.022 & -0.053 & -0.247 & -0.062 & 0.192 & 0.127 \\
\hline & Simulation (skewness) & 1.525 & 0.810 & 0.932 & 1.074 & 0.754 & 2.144 & 0.680 & 1.004 & 2.269 & 0.773 & 1.841 & 0.871 \\
\hline & Measured & 0.061 & 0.117 & 0.113 & -0.079 & -0.086 & 0.048 & -0.157 & 0.306 & -0.403 & 0.053 & -0.033 & -0.067 \\
\hline \multirow[t]{2}{*}{$r_{1, \tau}$} & simulation (normal) & 0.039 & 0.161 & 0.199 & -0.066 & -0.032 & 0.073 & -0.126 & 0.298 & -0.421 & 0.100 & 0.105 & 0.029 \\
\hline & Simulation (skewness) & 0.089 & 0.126 & 0.136 & -0.106 & -0.081 & 0.156 & -0.091 & 0.353 & -0.301 & 0.021 & -0.037 & -0.121 \\
\hline
\end{tabular}

Table 2 and Table 3 showed that the average, standard deviation, coefficient of skewness and first autocorrelation coefficient of the skewness sequence all well maintained for the measured one. However the standard deviation and the first order autocorrelation coefficient could be maintained and the other parameters varied greatly for the measured sequences, such as the average of simulated precipitation in September deviated larger from the measured one (see Figure 1), and the coefficient of normal sequence deviated largest from the measured one (see Figure 2). The results showed that the normal and the measured precipitation sequences didn't come from the same total. But the parameters of normal evaporation sequence remain good, except the coefficient of skewness. 
Table 3. Results of model suitability testing of Yuncheng district's monthly Evaporation

\begin{tabular}{|c|c|c|c|c|c|c|c|c|c|c|c|c|c|c|}
\hline \multicolumn{3}{|c|}{ Parameter } & \multicolumn{12}{|c|}{ monthly evaporation (mm) } \\
\hline & & & 1 & 2 & 3 & 4 & 5 & 6 & 7 & 8 & 9 & 10 & 11 & 12 \\
\hline \multirow[t]{3}{*}{$\bar{x}$} & measured & & 50.5 & 87.2 & 155.8 & 195.6 & 256.6 & 316.0 & 285.6 & 258.8 & 171.3 & 127.5 & 74.1 & 50.9 \\
\hline & Simulation & (normal) & 49.8 & 87.9 & 157.4 & 194.1 & 258.0 & 319.8 & 290.8 & 264.3 & 172.9 & 129.7 & 74.7 & 51.6 \\
\hline & Simulation & (skewness) & 50.2 & 86.2 & 156.3 & 197.0 & 256.6 & 319.5 & 285.6 & 253.1 & 169.8 & 124.7 & 74.8 & 51.7 \\
\hline \multirow[t]{4}{*}{$\sigma_{\tau}$} & measured & & 13.2 & 21.0 & 38.5 & 29.0 & 63.6 & 73.8 & 68.9 & 70.5 & 44.4 & 36.2 & 18.0 & 11.4 \\
\hline & Simulation & (normal) & 12.8 & 21.1 & 35.8 & 28.0 & 64.8 & 72.4 & 64.3 & 73.1 & 44.4 & 35.7 & 19.6 & 11.9 \\
\hline & Simulation & (skewness) & 13.7 & 19.4 & 37.7 & 30.7 & 64.5 & 76.3 & 65.1 & 73.7 & 42.8 & 36.3 & 19.8 & 10.9 \\
\hline & measured & & 0.471 & 0.965 & 1.375 & -0.172 & -0.134 & 0.240 & 0.233 & 0.776 & 0.126 & 0.306 & 0.509 & 0.633 \\
\hline \multirow[t]{3}{*}{ Cs } & Simulation & (normal) & 0.078 & 0.087 & -0.190 & -0.098 & 0.115 & 0.130 & 0.122 & -0.142 & 0.016 & 0.369 & -0.042 & 0.133 \\
\hline & Simulation & (skewness) & 0.681 & 0.696 & 1.590 & -0.024 & -0.338 & 0.285 & 0.282 & 1.044 & 0.238 & 0.592 & 0.576 & 0.510 \\
\hline & measured & & 0.219 & 0.375 & 0.328 & 0.109 & 0.293 & 0.567 & 0.295 & 0.417 & 0.335 & 0.634 & 0.237 & 0.391 \\
\hline \multirow[t]{2}{*}{$r_{1, \tau}$} & Simulation & (normal) & 0.190 & 0.439 & 0.347 & 0.078 & 0.376 & 0.564 & 0.327 & 0.373 & 0.332 & 0.613 & 0.239 & 0.408 \\
\hline & Simulation & (skewness) & 0.124 & 0.325 & 0.324 & 0.131 & 0.316 & 0.530 & 0.293 & 0.374 & 0.413 & 0.678 & 0.372 & 0.373 \\
\hline
\end{tabular}

In addition, negative values were found in the simulated sequence of precipitation regardless of the normal or skewed distribution. It did not match the actual situation. Thus statistic was done for the sum of the negative number and negative value in three groups of 100 length simulated sequences. The results were shown in table 4 . It also could be seen from table 3 that not only the number of negative in the skewness sequence was less than in the normal one, decreased by nearly $50 \%$, but also the negative sum in the skewness sequence was much smaller than the normal one, decreased by nearly $90 \%$. The result showed that precipitation sequence should be simulated by the skewness model.

Table 4. Statistics of the number and the negative sum of the negative in the simulated precipitation sequence

\begin{tabular}{|c|c|c|c|c|c|c|c|c|}
\hline \multirow{2}{*}{ item } & \multicolumn{4}{|c|}{ Simulation (normal) } & \multicolumn{4}{|c|}{ Simulation (skewness) } \\
\hline & sample1 & sample2 & sample3 & average & sample1 & sample2 & sample3 & average \\
\hline $\begin{array}{c}\text { Number of the } \\
\text { negative }\end{array}$ & 150 & 138 & 168 & 152 & 67 & 66 & 93 & 75 \\
\hline $\begin{array}{c}\text { Sum of the } \\
\text { negative }\end{array}$ & -2676.5 & -2800.7 & -2776.4 & -2751.2 & -274.9 & -274.6 & -439.1 & -329.5 \\
\hline
\end{tabular}




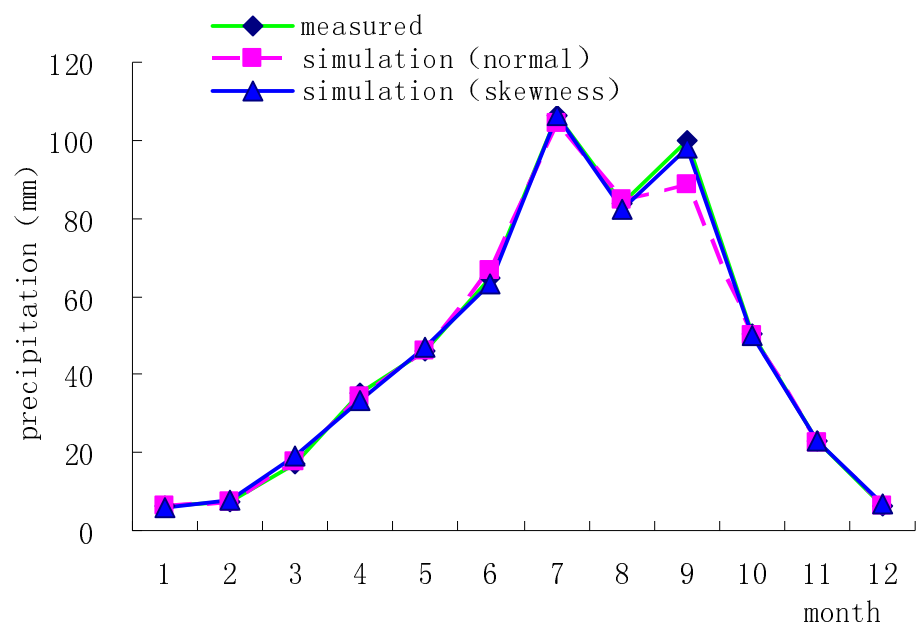

Fig. 1. Comparison of the average of precipitation simulated and measured sequences

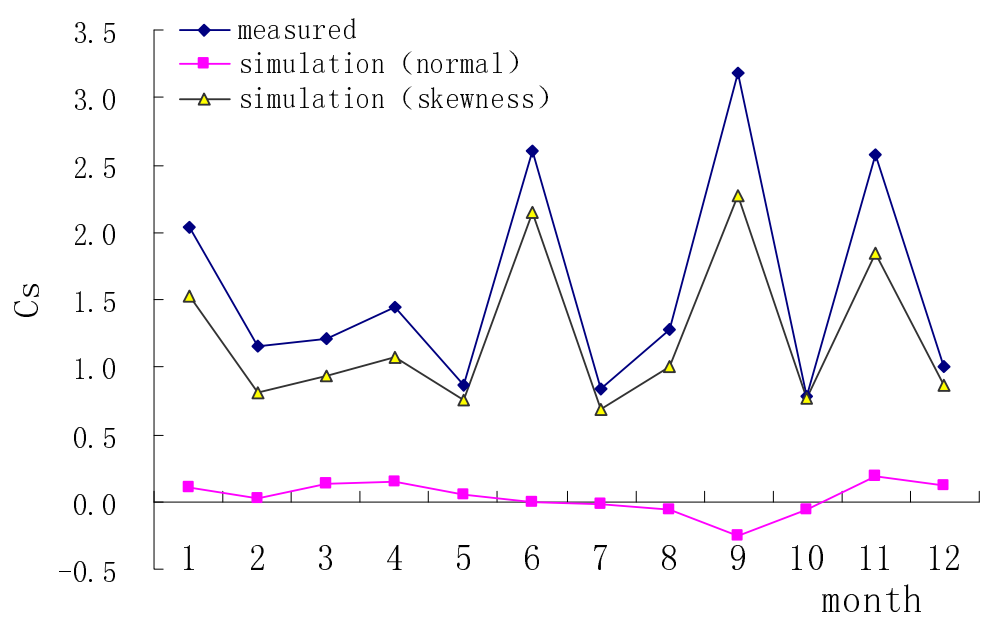

Fig. 2. Comparison of the coefficient of skewness of precipitation simulated and measured sequences

\subsection{Analysis of Irrigation Water's Frequency Curve}

Through the skewness model, 20 groups of equal length with the measured sequence of precipitation and evaporation sequence can be generated. The sequence of irrigation water of the winter wheat multiple corns planting type had been calculated using the formula (12). Then based on the measured sequence of precipitation and evaporation, this paper calculated the measured irrigation water sequence using 


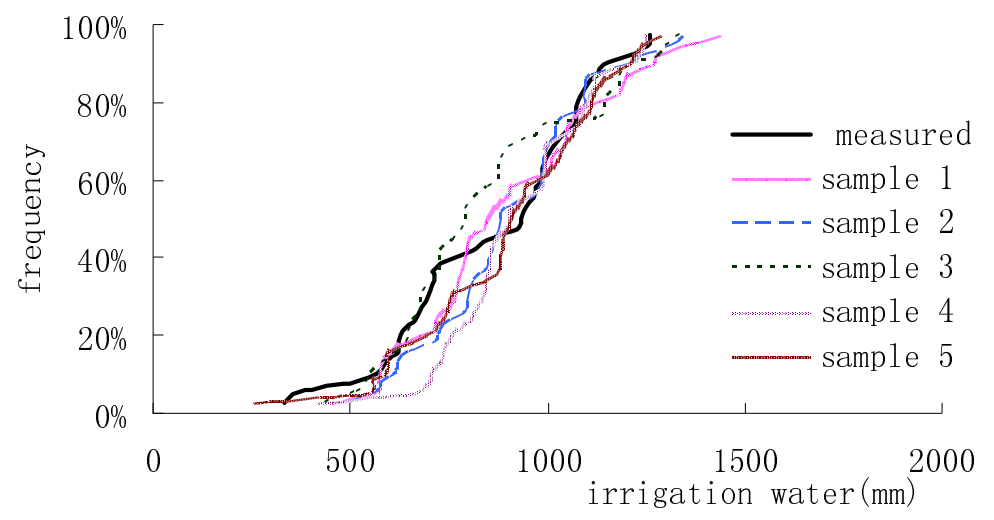

Fig. 3. The frequency curves of irrigation water

the " $\alpha$ " value method. Statistic was done for the data of irrigation water by frequency calculation method. Finally, five simulation groups of irrigation water's frequency curves were compared with the measured one. The results were shown in figure 3.

Figure 3 showed that simulated frequency curves fit the measured one good. 20 groups of equal length with the measured sequence of simulated irrigation water were compared with the measured one. The results were shown in table 5 and 6 . As can be seen from table 5, the average、standard deviation and coefficient of skewness of simulated sequences well maintained for the measured one. Table 6 showed that the differences between simulated irrigation water sequences and measured one were little, and the difference between the maximum and minimum values was from 100 to $200 \mathrm{~mm}$. If approximatively taking the average of 20 simulated groups as the average of total, the measured values of irrigation water were lager than the total's average between the $50 \%$ and $90 \%$ frequencies. Thus if the scale of irrigation projects was determined using the measured sequence, the result could be larger than the actual.

Table 5. Statistical parameters of irrigation water simulation sequences and measured values sequences

\begin{tabular}{cccc}
\hline & $\bar{x}$ & $\sigma_{\tau}$ & $C_{s}$ \\
\hline measured & 863.6 & 242.1 & -0.333 \\
simulation average & 865.9 & 217.8 & -0.306 \\
\hline
\end{tabular}

Table 6. Comparison of measured and simulated values at different frequencies (mm)

\begin{tabular}{cccccc}
\hline frequency & measured & simulation & $\max$ & $\min$ & max-min \\
\hline $25 \%$ & 670 & 717 & 810 & 636 & 174 \\
$50 \%$ & 932 & 883 & 942 & 824 & 118 \\
$75 \%$ & 1069 & 1027 & 1086 & 944 & 142 \\
$90 \%$ & 1141 & 1134 & 1205 & 1036 & 169 \\
$95 \%$ & 1224 & 1256 & 1315 & 1122 & 193 \\
\hline
\end{tabular}




\section{Conclusions}

Precipitation and evaporation of Yuncheng were simulated by the seasonal autoregressive model, which considered separately for the normal and skewed distribution of precipitation and evaporation. Long series of monthly precipitation and evaporation sequences were generated. The simulation results showed that the skewed simulation had better agreement with the measured. Then based on the data of skewed distribution, this paper established the relations formula of water evaporation and crop water requirement, and calculated the crop water requirement. The long series of crop irrigation water were obtained through the water balance calculation. Comparing with the measured irrigation water, it verified the practicability of the model. The results showed that the model was feasible in allowable error.

Acknowledgment. Funds for this research was provided by the National Science and Technology Support Program (2012BAD08B01).

\section{References}

[1] Azhar, A.H., Murty, V.V.N., Phiem, H.N.: Modeling irrigation schedules for lowland rice with stochastic rainfall. Journal of Irrigation and Drainage Engineering 118(1), 36-55 (1992)

[2] Cai, H.: Stochastic simulation of Precipitation and its application in irrigation. Irrigation and Drainage 10(2), 8-14 (1991)

[3] Zuo, X.: Irrigation process of paddy based on random simulation of precipitation and evaporation. Hohai University, 6 (2005)

[4] Wen, J., Guo, S., Guo, D.: Stochastic simulation model for winter wheat irrigation. Transactions of the CSAE 21(11), 25-28 (2005)

[5] Zai, S.-M., Wen, J., Guo, D.-D.: Stochastic Simulation for Crop Water Requirement. Journal of Irrigation and Drainage 28(5), 92-95 (2009)

[6] Wen, J., Guo, S., Lu, W.: Research of Stochastic Simulation Technology of Crop Irrigation. Yellow River 26(5), 39-41 (2004)

[7] Ding, J., Liu, Q.: Stochastic hydrology, pp. 17-27. China Water Power Press, Beijing (1997) 